\title{
Réflexions sur la formation en gammagraphie et radiographie industrielle
}

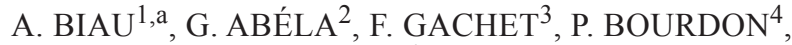 \\ J.-C. LEQUEUX ${ }^{4}$, E. MARTIN ${ }^{5}$, \\ S. RETKOWSKI ${ }^{6}$, P. ROINE ${ }^{7}$, J.-P. SERVENT ${ }^{8}$, V. SOARES ${ }^{9}$, T. VIAL $^{10}$
}

RÉSUMÉ Comme pour nombre de métiers la formation débute par un apprentissage avec un formateur ou compagnon pour connaître les gestes, les postures, les actions qui concourent à des prestations de qualité dans des conditions optimales de sécurité. Pour la manipulation d'appareils de radiographie industrielle (rayons $X$ ou gamma) une connaissance spécifique supplémentaire doit être acquise dans le domaine des risques liés à l'utilisation des rayonnements ionisants. Afin de limiter ces risques pour les opérateurs et pour le public en général toute une batterie de textes réglementaires a été établie en droit français. Ces textes concernent la détention de sources de rayonnements ionisants, le transport de ces sources, le contrôle des appareils, la formation et le suivi dosimétrique des travailleurs (voir les travaux de l'atelier 1). Tous ces textes ont permis en France de comptabiliser peu ou pas d'incidents graves durant les deux dernières décennies, si l'on excepte le cas particulier de Forbach en 1991 dont l'accélérateur d'électrons était utilisé pour des irradiations mais qui aurait pu être utilisé en radiographie. Toutefois des incidents mineurs ont toujours lieu et la moyenne des doses individuelles des opérateurs de radiographie, notamment en gammagraphie, reste une des plus élevées de l'ensemble des travailleurs en France. La meilleure façon de limiter encore l'exposition de ces travailleurs est d'améliorer la formation tant sur le plan de la radioprotection en général que sur celui des bonnes pratiques professionnelles, les deux étant d'ailleurs intimement liés. Le but de cet atelier a été de faire le point sur les aspects réglementaires de la formation en radioprotection et voir comment la faire évoluer pour limiter les expositions des intervenants en radiologie industrielle. La première partie de ce document expose les réflexions de l'atelier à partir de la fin de l'année 2006 et la deuxième partie présente les deniers textes réglementaires du 21 décembre 2007 et détaille les principales dispositions prises par rapport aux propositions de l'atelier. 
As for all the trades the formation begin with a training with a trainer or companion to know the gestures, postures, the actions which contribute to services of quality under optimum conditions for safety. For the handling of apparatuses of industrial radiography (X-ray or gamma) an additional specific knowledge must be acquired in the field of the risks related to the use of the ionizing radiations. In order to limit these risks for the operators and the public in general a whole battery of lawful texts was established in French right. These texts relate to the detention of ionizing radiation sources, the transport of these sources, the control of the apparatuses, the training and the dosimetric follow-up of the workers (see work of the workshop 1). All these texts made it possible in France to enter little or not serious incidents during the two last decades, if one excludes the particular case of Forbach in 1991 whose accelerator of electrons was used for irradiations but which could have been used in radiography. However minor incidents always take place and the average of the personal doses of the operators of radiography, in particular in gammagraphy, remains one of highest of the whole of the workers in France. The best way of still limiting the exposure of this type of workers is to improve the formation as well in the field of protection against radiation in general as on that of the good professional practices, both being besides closely dependent. The goal of this workshop was to give a progress report on the lawful aspects of the training in protection against radiation and to see how to make it evolve/ move to limit the exposures of the workers in industrial radiography. The first part of this document presents the reflexions of the workshop starting from the end of the year 2006 and the second part presents the lawful sums of money texts of December 21, 2007 and details the principal provisions taken compared to the proposals of the workshop.

\section{La formation en radioprotection dans le domaine de la radiologie industrielle}

\subsection{Aspects règlementaires de la formation}

\subsubsection{CAMARI}

L'article R-231-91 du code du travail impose l'obtention du Certificat d'aptitude à manipuler des appareils de radiologie industrielle (CAMARI), l'arrêté d'application correspondant, du 25 juin 1987, était en cours de réécriture lors de travaux de l'atelier. Le nouvel arrêté a été finalisé le 21 décembre 2007 et édité au Journal officiel du 28 décembre 2007.

Sur la forme, une modification importante était prévue dans le mode d'attribution de ce certificat qui faisait l'objet d'un examen régional dans les directions régionales du travail et de l'emploi. La nouvelle version devrait imposer un examen national pour éviter d'éventuelles hétérogénéités dans l'appréciation des connaissances nécessaires à ce type de travail. L'atelier s'est montré très favorable à cette modification sans préjuger de l'organisation effective de ce contrôle de connaissance.

Sur les modalités, cet examen doit comprendre une épreuve écrite sur les connaissances de base concernant la nature des rayonnements ionisants, les 
grandeurs dosimétriques, les principes de base de la radioprotection et les risques associés aux expositions accidentelles ou chroniques.

Une épreuve orale à la suite d'un stage pratique en entreprise constituera le deuxième volet de cet examen.

Lors de ce stage pratique l'opérateur devra notamment apprendre l'utilisation du matériel radiologique, des dosimètres actifs et passifs et des radiamètres pour vérifier le bon fonctionnement du matériel et délimiter les zones d'exclusion.

Sur le champ d'application, l'article R 231-91 impose à tout travailleur de posséder ce certificat d'aptitude (CAMARI) pour pratiquer des radiographies industrielles avec des sources radioactives (gammagraphie) ou avec des appareils émetteurs de rayons $X$. La liste des appareils dont l'utilisation nécessite le CAMARI devra être fixée par arrêté.

Concernant les connaissances de base nécessaires pour ce type d'activité professionnelle et compte tenu de la variété des niveaux d'études des candidats à cet examen, un haut niveau scientifique dans le domaine de la physique nucléaire n'est pas indispensable mais il faut connaître de façon sûre tous les principes de radioprotection élémentaire et la nature des risques liés à l'exposition aux rayonnements ionisants ainsi que l'essentiel des aspects règlementaires.

À ce titre le candidat doit connaître parfaitement les lois principales de la radioprotection concernant l'exposition externe, seule concernée en radiologie industrielle, en fonction des paramètres temps, distance et épaisseur d'écrans :

\section{- Loi linéaire en fonction du temps}

La dose reçue en un point où le débit de dose est $D$ est proportionnelle au temps $t$ passé en ce point.

Cela paraît évident mais il faut insister sur cet aspect basique de radioprotection : moins l'opération dure et moins la dose est élevée mais comme il ne faut pas confondre "vitesse et précipitation » il faut bien étudier au préalable le protocole d'intervention de façon à être efficace et par exemple éviter la multiplication des tirs radiographiques qui augmentent l'exposition de l'opérateur et le coût de l'opération.

\section{- Loi de l'inverse du carré de la distance}

Si le débit de dose à $1 \mathrm{~m}$ d'une source ponctuelle est $D$, à la distance $d$ il est de $D / d^{2}$.

Quelques petits calculs à faire de tête montrent que la dose augmente très vite lorsqu'on s'approche de la source et a fortiori lorsqu'on la touche (tous les accidents graves ou mortels de gammagraphie ont eu lieu après contact direct avec 
la source) alors qu'à grande distance (plus de 10 m) un écart de $1 \mathrm{~m}$ n'entraîne qu'une faible variation de la dose.

Il faut bien avoir en tête certains repères : à $1 \mathrm{~m}$ dose $D$, à $10 \mathrm{~cm} D \times 100$, à $1 \mathrm{~cm} D \times 10000$, à $10 \mathrm{~m} D / 100$.

En pratique il faut donc essayer de se tenir le plus loin possible de la source tout en dominant la situation (utilisation possible de pinces à distance, télécommande, etc.).

\section{- Loi exponentielle, décroissance radioactive et atténuation dans les matériaux}

La loi exponentielle s'applique à la décroissance radioactive en fonction du temps et à l'atténuation du rayonnement $\mathrm{X}$ ou $\gamma$ en fonction de l'épaisseur de matériaux traversée.

En radiographie $\mathrm{X}$ le problème de la décroissance radioactive avec le temps ne se pose pas et pour la gammagraphie avec l'iridium-192 la décroissance est négligeable sur la durée d'une opération.

En revanche l'atténuation dans les matériaux est fondamentale en termes de radioprotection. Si on dispose d'écrans (par exemple de plomb) d'épaisseur $x$ qui atténuent le rayonnement d'un facteur $a$, une épaisseur de $2 x$ atténue d'un facteur $a^{2}$, une épaisseur $3 x$ d'un facteur $a^{3}$ et ainsi de suite en première évaluation.

Pour le calcul mental il est facile d'utiliser les couches de demi atténuation (CDA) c'est-à-dire l'épaisseur d'un matériau qui atténue d'un facteur 2 les rayonnements $\gamma$ d'énergie donnée, ainsi une CDA atténue d'un facteur 2, deux CDA d'un facteur $2^{2}=4$, trois CDA d'un facteur $2^{3}=8$ et ainsi de suite. À retenir, pour dix CDA l'atténuation est de 1024 soit $\sim 1000$.

La bonne connaissance de ces lois permet de connaître les ordres de grandeur essentiels nécessaires pour l'étude du poste de travail mais il faut conforter ces évaluations par des mesures avec des radiamètres pour déterminer les doses réelles tenant compte d'autres paramètres tels que le rayonnement diffusé, le build up factor, etc.

\subsubsection{Formation du personnel exposé}

L'article R 231-89 du code du travail (décret du 31 mars 2003) impose une formation à la protection contre les rayonnements ionisants pour les agents susceptibles d'intervenir en zone surveillée ou contrôlée tous les trois ans.

Cette formation organisée par le chef d'établissement porte sur :

- les risques liés à l'exposition aux rayonnements ionisants ;

- les procédures de radioprotection mis en œuvre dans l'établissement adaptées au poste de travail ; 
- les règles de conduite en cas de situations anormales ;

- les règ les particulières concernant les femmes enceintes.

Cette formation concerne directement les travailleurs titulaires du CAMARI, il ne s'agit donc pas de refaire la formation décrite au paragraphe 1.1.1. mais de faire des rappels en insistant sur les spécificités propres à l'entreprise, les innovations techniques et autres évolutions professionnelles.

\subsection{Aspects pratiques de la formation professionnelle}

\subsubsection{Les bons gestes}

Les bons gestes découlent de l'expérience transmise par les gens du métier aux nouveaux arrivants. Ils doivent être décrits dans des modes opératoires établis par la profession tout en donnant une certaine latitude pour faire face à des conditions imprévues, topographie complexe, lieu public, etc.

En tout état de cause le bon geste est celui qui concilie les objectifs de qualité de la prestation, qualité du cliché en fonction de l'angle de tir, de la distance, de la nature de l'objet à radiographier et les impératifs de radioprotection pour l'agent lui-même et son environnement, sans oublier les contingences de sécurité classiques, travail de nuit, port de charges, travail sur échafaudages...

\subsubsection{La gestion des incidents}

D'une manière générale lors d'une situation imprévue il faut d'abord raisonner en termes de radioprotection et agir de façon à éviter ou tout au moins limiter l'exposition des opérateurs et du public éventuel.

La base RELIR donne quelques exemples d'incidents réels survenus en France qui montrent ce qu'il faut faire et ne pas faire, ces incidents ont été choisis pour leur aspect pédagogique (voir atelier 2).

Pour réagir rapidement et efficacement dans les situations d'incidents les plus courants il est nécessaire d'avoir des fiches réflexe simples qui précisent les actions à mener pour limiter les conséquences de ces situations.

Le retour d'expérience sur les trente dernières années montre que les incidents les plus caractéristiques sont :

- panne du véhicule,

- accident de circulation du véhicule transportant la source,

- vol du véhicule,

- source bloquée dans sa gaine d'éjection,

- erreur de procédure, 
- alarme d'un dosimètre actif,

- source désolidarisée du porte-source,

- découverte d'un intrus dans la zone d'exclusion,

- perte d'un dosimètre actif ou passif.

L'atelier 2 expose plus précisément les différents types d'incidents et les conduites à tenir mais trois types d'incidents méritent une attention particulière.

\section{Vol de véhicule transportant l'appareil de gammagraphie}

En général l'objet du vol est le véhicule lui-même mais lorsque le voleur découvre l'appareil il peut soit le jeter sur la voie publique (risque de démontage par n'importe qui et libération de la source), soit le vendre à un ferrailleur, c'est comme cela qu'on a retrouvé du cobalt-60 dans des armatures de béton ou des axes de bracelet de montres!

Il ne faut pas non plus écarter la possibilité d'agression pour utiliser la source pour des raisons malveillantes.

Il faut donc ne jamais laisser sans surveillance le véhicule tant que l'appareil est à l'intérieur et il faudra envisager que les appareils soient dotés de « puces » de façon à ce qu'ils soient repérables à distance si malgré toute la surveillance il y a vol par braquage par exemple.

\section{Source désolidarisée et perdue sur un chantier}

C'est encore plus grave puisque c'est ce type d'incident qui peut gravement blesser ou tuer si quelqu'un récupère cette source, la met dans sa poche et la ramène chez lui comme cela a été le cas encore récemment au Chili.

Avec du matériel correctement entretenu et une bonne organisation du travail le risque est heureusement faible. Il faut malgré tout rester très vigilant.

\section{Intrusion d'une personne dans la zone d'exclusion}

Ce type d'incident fait en général beaucoup de bruit car il dénote un défaut d'organisation et soumet à une exposition potentielle des personnes a priori non exposées mais il est rare que l'intrusion dure plus d'une minute à 10 mètres de la source. Un calcul rapide montre que la dose reçue éventuellement reste très inférieure à $1 \mathrm{mSv}$. Si les conséquences objectives sont faibles voire négligeables, ce type d'incident demeure inacceptable.

\subsubsection{Problèmes liés à la manipulation d'appareils lors de la formation initiale}

La réglementation exigeant le CAMARI pour la manipulation des appareils de radiologie industrielle, le problème de l'apprentissage se pose pour les candidats à l'obtention de ce certificat. 
Cette difficulté de fond a été posée notamment par l'Institut de formation et assistance technique (IFAT) qui est confronté à cette contradiction entre la nécessité du CAMARI pour manipuler et celle de manipuler pour obtenir le CAMARI.

Il est attendu que l'arrêté précise les conditions de manipulation et d'encadrement des candidats au CAMARI de façon à ce que l'aspect pratique de la formation soit effectif et validé par des personnes compétentes.

\section{Les derniers textes règlementaires}

Deux arrêtés du 21 décembre 2007 sont parus au Journal Officiel du 28 décembre 2007. Le premier fixe la liste des appareils dont la manipulation nécessite l'obtention du CAMARI, et le second définit les modalités de formation et de délivrance du CAMARI.

Un examen rapide de ces deux textes règlementaires permet de vérifier que l'essentiel des réflexions de l'atelier se trouve pris en compte.

\subsection{Arrêté du 21 décembre 2007 portant homologation de la décision $n^{\circ} 2007$ DC 0074 \\ de l'Autorité de sûreté nucléaire du 29 novembre 2007 fixant \\ la liste des appareils ou catégories d'appareils pour lesquels \\ la manipulation requiert le certificat d'aptitude mentionné \\ au premier alinéa de l'article R.231-91 du code du travail}

Cet arrêté ouvre le champ des activités nécessitant la certification CAMARI aux appareils à rayons $\mathrm{X}$ fonctionnant sous une différence de potentiel supérieure à $200 \mathrm{kV}$ et aux accélérateurs de particules d'énergie supérieure à $1 \mathrm{MeV}$ à l'exclusion de ceux utilisés à des fins de recherche.

Jusqu'à la parution de cet arrêté, le CAMARI était obligatoire pour les appareils contenant au moins une source radioactive et utilisés en radiologie industrielle. Il l'est aussi désormais pour les appareils mobiles d'étalonnage contenant « au moins une source radioactive de haute activité, qu'ils soient utilisés ou non à poste fixe $»$.

Sont exclus :

- les appareils émetteurs de rayons X qui, en fonctionnement normal, ne créent en aucun point situé à $10 \mathrm{~cm}$ de leurs surfaces accessibles des débits d'équivalent de dose supérieurs à $10 \mu \mathrm{Sv} / \mathrm{h}$;

- les appareils émetteurs de rayons $\mathrm{X}$ à poste fixe dans une installation ne nécessitant pas la présence d'un opérateur à l'intérieur du local et dont, en 
fonctionnement normal, les débits d'équivalent de dose à $10 \mathrm{~cm}$ des surfaces accessibles ne sont pas supérieurs à $10 \mu \mathrm{Sv} / \mathrm{h}$;

- les appareils à usage vétérinaire ;

- les appareils de contrôle de bagage ;

- les appareils dispensés de l'autorisation ou de la déclaration prévue à l'article L.1333-4 du code de la santé publique.

\subsection{Arrêté du 21 décembre 2007 définissant les modalités de formation et de délivrance du Certificat d'Aptitude à Manipuler les Appareils de Radiologie Industrielle (CAMARI)}

Les modalités d'obtention du « nouveau » CAMARI sont définies essentiellement aux articles 2, 3 et 4, et le contenu pédagogique est défini en annexe 1.

\subsubsection{Modalités d'obtention}

- article 2 : «L'Institut de radioprotection et de sûreté nucléaire organise le contrôle des connaissances des candidats qui justifient d'une formation délivrée dans les conditions mentionnées à l'article 3 et dont le module pratique a été enseigné depuis moins d'un an ».

- article 3 : « La formation est dispensée selon trois options :

- générateur électrique de rayons X,

- accélérateur de particules,

- appareil de radiologie industrielle contenant, au moins, une source radioactive. »

Elle comprend deux modules dont le contenu et la durée sont définis en annexe :

- « un module théorique, relatif aux principes de la radioprotection et à la réglementation en matière de radioprotection, commun aux trois options,

- un module pratique, spécifique à chacune des options. »

- article 4: «La délivrance par l'Institut de radioprotection et de sûreté nucléaire du CAMARI est subordonnée à la réussite des épreuves de contrôle des connaissances qu'il a organisée et qui comprennent :

- une épreuve écrite portant sur les objectifs pédagogiques définis à l'annexe 1 . En cas de réussite il est remis par l'Institut de radioprotection et de sûreté nucléaire au candidat un certificat provisoire valable un an lui permettant de préparer l'épreuve orale ;

- une épreuve orale qui se déroule après une période probatoire d'au moins trois mois. ")

Le reste de cet article décrit les modalités de formation pratique à la manipulation des appareils de radiologie industrielle du candidat ayant obtenu le certificat provisoire cité ci-dessus :

«Ces manipulations s'effectuent sous la surveillance d'un professionnel titulaire d'un CAMARI en cours de validité responsable des opérations liées 
à la mise en œuvre de l'appareil. En outre, le candidat participe à toutes les opérations nécessaires à la mise en ouvre de cet appareil et des mesures de prévention appropriées, par exemple le balisage, la maintenance, l'entreposage et le transport. »

À l'issue de cette période le candidat rédige un rapport transmis à l'IRSN avant l'épreuve orale.

En cas de succès le CAMARI est délivré pour une période de cinq ans.

\subsubsection{Contenu pédagogique}

L'annexe 1 ce cet arrêté précise le contenu pédagogique de la formation qui doit permettre au candidat « d'acquérir les compétences en matière de radioprotection nécessaires à la prévention du risque radiologique associé à l'appareil qu'il met en œuvre. »

\section{Le module théorique comprend trois unités :}

Unité 1 : rayonnements ionisants et effets biologiques (radioactivité, production des rayonnements, l'interaction des rayonnements avec la matière, les effets biologiques et les sources d'exposition).

Unité 2 : radioprotection des travailleurs (protection contre le rayonnement externe, la détection des rayonnements et les calculs simples de débit de dose et de protection).

Unité 3 : réglementation (les principes de radioprotection, justification, optimisation et limitation, la protection des travailleurs, les conditions de délimitation des zones d'opération, la surveillance de l'exposition des travailleurs et le rôle de la personne compétente en radioprotection).

Ce module théorique doit durer au moins 16 heures.

\section{Le module pratique comprend les objectifs suivants :}

- connaître les règles de sécurité applicables aux familles d'appareils de l'option choisie,

- connaître les moyens de suivi dosimétrique (passif et opérationnel),

- établir la délimitation et la signalisation de la zone d'opération,

- utiliser les appareils de détection,

- gérer une situation radiologique dégradée ou accidentelle.

La durée effective de ce module est au moins égale à 16 heures. 


\section{Conclusions}

Les textes des arrêtés décrits ci-dessus sont globalement conformes aux réflexions menées dans l'atelier 3 .

En particulier les modalités de la formation pratique, notamment la manipulation des appareils encadrée par un titulaire du CAMARI, sont plus claires et répondent aux demandes des formateurs.

L'examen normalisé au niveau national sous l'égide de l'IRSN était également souhaité, reste à concrétiser la mise en application de cette nouvelle disposition.

Sur le contenu pédagogique, la volonté de s'appuyer sur des connaissances élémentaires et simples mais solidement acquises est tout à fait dans la ligne de ce qui a été discuté dans l'atelier. 